\title{
GA based Automatic Optic Disc Detection from Fundus Image using Blue Channel and Green Channel Information
}

\author{
G.Ferdic Mashak Ponnaiah, \\ Research Scholar, Dept of Computer \\ Science and Engineering,Bharathiar, \\ University, Coimbatore
}

\author{
S.Santhosh Baboo, PhD. \\ Associate Professor, PG \& Research Center \\ Dept of Computer Science,D.G.Vaishnav \\ College, Arumpakkam, Chennai
}

\begin{abstract}
Proper detection of Optic disc (OD) in a fundus image is an important stage in an automated method to screen diabetic retinopathy. To identify the OD the intensity of the pixel is used. The pixel of the OD is different from the rest of the fundus image and intensity based techniques such as thresholding may be applied for the detection. Due to more brighter region false resemblens of OD is generated and the intensity based technique fails to detect the OD properly. Using techniques like template matching the OD is detected [1] and [2]. The sliding window tehnique applied in template matching is time consuming. The method proposed is using Genetic Algorithm which will search the entire fundus image in a short time.

Almost in every intensity based method, the red channel and green channel informations are used. The Blue channel information is neglated and the reason for avoiding is no potential information in the blue channel, which is not true. Since we are detecting the OD and not the Exudates or other symptoms the blue channel will be suitable for a dull image.In this work, we tried to use the blue layer information as the major clue for the successful detection of optic disc location and size in a typical fundus image. We designed a genetic algorithm based algorithm as well as a direct search based algorithm to locate the exact position and size of the OD using blue layer and green layer intensity in a suitable fitness function.
\end{abstract}

Index Terms - Diabetic Retinopathy, Optic Disc Detection, Nonlinear Optimization.

\section{INTRODUCTION}

Diabetic retinopathy is a serious problem and mostly it affects people who are having diabetes for a long time. The working age group is mostly affected by diabetic retinopathy and lead them to blindness. This can be controlled by regular screening of the fundus image for any disorder in the retinopathy. To screen the increasing volume of patient an automated method is needed. The method may use the digital image of the fundus and generate a report of abnormalities. The detection of fundus image structures such as Optic Disc,blood vessels are important to identify the symptoms such as Hard exudates, Soft exudates etc.Optic disc
(OD) detection is a first and foremost main step while developing automated method to screen the diabetic retinopathy. Optic disc boundary and localization of macula are the two features of retina were necessary for the detection of exudates and also knowing the severity of the diabetic maculopathy[4].

\subsection{Fundus Image in Retinopathy}

Fundus imaging is an eye clinical procedure used for viewing the retina and keep a record of the patient. This help for diagnosis, treatment process and evaluation of the patient. The fundus images are captured using fundus camera which is a low power speialized microscope. Fundus photography is used to generate a document of diabetic retinopathy characteristics such as microaneurysms and macular edema. Because retinal details are more easier to visualize in stereoscopic fundus photographs compare with direct examination.

\subsection{Optic Disc (OD)}

In the eye the OD is the end point of retinal nerve. OD is a brighter region when compare with the rest of the ocular fundus and its shape is usually round. The location of OD is an essential process in fundus image analysis to locate anatomical and pathological parts in retinal images.Detection of optic disc used to identify diseases such as glaucoma and the development of new blood vessels which is very dangerous.

The central portion of disc is the brightest region called optic cup or optic disc.The blood vessels and nerve fibers are absent in optic disc.

\section{PROBLEM DEFINITION}

The localization of optic disc is important for several reasons. The OD location serves as the baseline for finding the exact boundary of the disc[2]. Optic disc center and diameter are used to locate the macula in the image. In some methods and practices, OD location of the fundus image is used as a reference point or registration point. Further, false detections at the OD regions should be eliminated from the calculations of accuracy for that the exact region of OD should be known. 
It is important to detect and isolate OD region since most of the algorithms designed to detect abnormalities such as Hardexudates will detect lot of false positives in OD region. In other words, most of the pixel intensity and colour based abnormality detection algorithms will detect OD region as a abnormality. So, the false positives in the OD region should be eliminated from the calculations of accuracy of a abnormality detection algorithm.

In a colour retinal image the optic disc belongs to the brighter parts along with some lesions. Applying a threshold may separate part of the optic disc and some other unconnected bright regions from the background[4]. How ever, further processing will be needed to separate the OD region alone from the image. So, the detection of exact location of OD is an important task and it is still a challenging task.

In [1], Tomi Kauppi and Heikki Kälviäinen proposed a method that utilizes template matching and PCA(Principal component Analysis) which have been used for optic disc extraction. Li and Chutatape [2] applied the PCA based face detection method originally proposed by Turk and Pentland [3] for optic disc extraction. In this work, Tomi Kauppi and Heikki Kälviäinen's work as well as $\mathrm{Li}$ and Chutatape's method are used as a comparison methods. We decided to compare these two works with our proposed optic disc detection method because, these two works are considered as a baseline work since these works are refered by Tomi Kauppi, who is the main contributor of DIARETDB1.

\section{DETECTION OF OD USING NONLINEAR OPTIMIZATION}

In most of the OD detection methods, the entire image is searched for finding a matching OD profile. This search will be accomplished by the sliding window operation. The sliding window operation will evaluate almost all the pixel location of the eye fundus image for the detection of OD. But it is possible to use a nonlinear optimization technique to reduce the time needed sliding window operation.

Most of the real world system models involve nonlinear optimization with complicated objective functions or constraints for which analytical solutions are not available. In such cases one of the possible solutions is the search algorithm in which, the objective function is first computed with a trial solution and then the solution is sequentially improved based on the corresponding objective function value till convergence.

So, we can use a suitable objective function for finding the optimum location of OD in the entire image. Flowchart of the nonlinear search algorithm with decision variable $\mathrm{Xi}$, is given in the following flow chart.

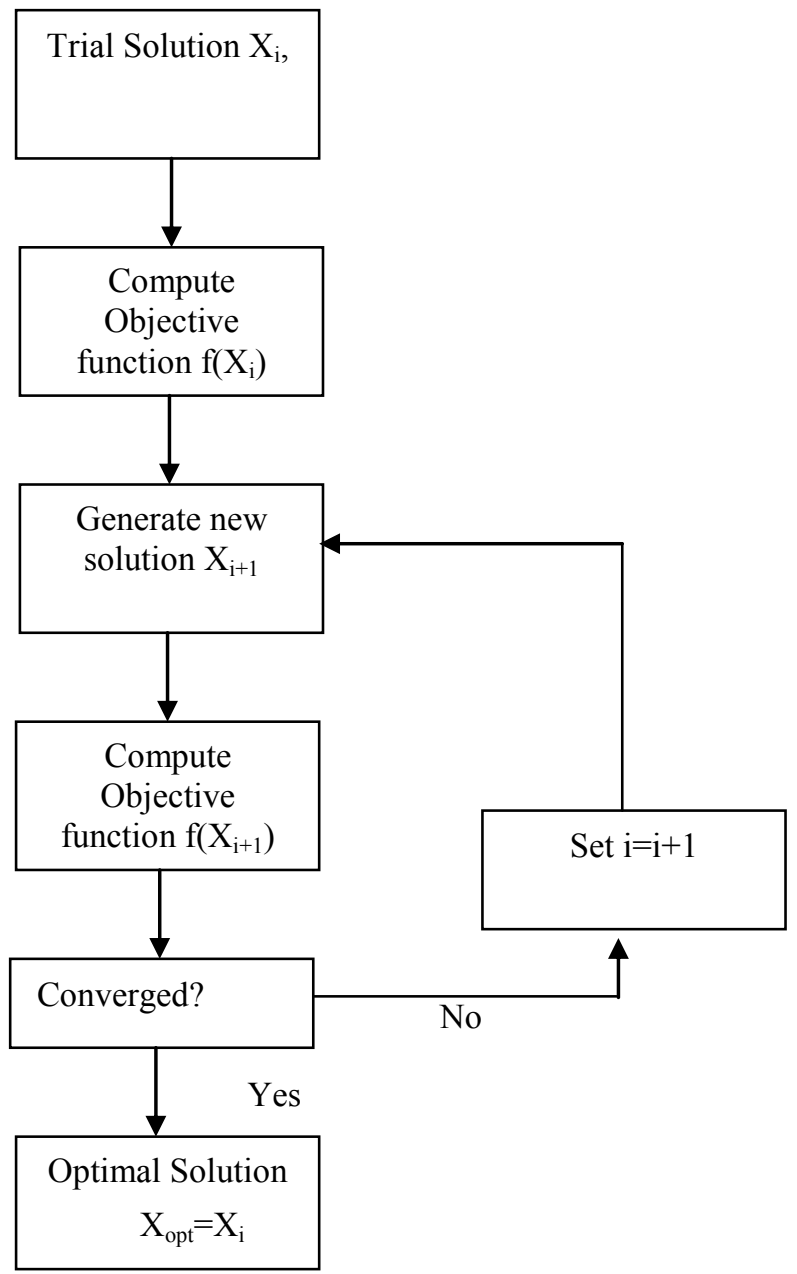

\subsection{Genetic Algorithm}

The Genetic algorithm consist of the important components such as Representation, Fitness, Population, Parent selection,Variation operators and Replacement

\subsubsection{Representation}

Representation is a process of mapping of phenotypes on a set of genotypes. Phenotypes are objects forming possible solution within the original proplem. The phenotype encoding, with the individuals within the GA, are called genotypes.

\subsubsection{Variation Operators}

The function of variation operator is creating new individuals from old ones. The elementary step within the search operation is done by variation operator. 


\subsubsection{Mutation Operator}

Mutation is a genetic operator used to maintain genetic diversity from one generation to another generation.It is applied to one genotype and delivers a modified mutant. A random unbiased change is caused by mutation and space connection is guaranteed.

\subsubsection{Crossover Operator}

Process of taking more than one parent solutio and deliver a child solution from them.

\subsubsection{Parent Selection Mechanism}

Based on the better individuals quality to become the next generation parent.

\subsubsection{Survivor Selection Mechanism}

In each successive generation a portion of the existing population is selected to bread new generation. The parent selection is stochastic, survivor selection is deterministic.

\subsubsection{Initialization}

- Initialization can be done by selecting the population randomly. Population size depends on the nature of the problem

- Can be applied or seeded in areas where optimal solution likely to be found.

\subsubsection{Termination Condition}

- Solution found which satisfies the criteria.

- Fixed number of generation reached.

- Allocated budget(time/money) reached.

- The highest ranking solution method.

- Combination of the above.

\subsubsection{Population}

Population is to hold possible solutions and it is a multiset of genotypes. The size of population is constant and not changing while the evolutional search.

\section{EVALUATION OF ALGORITHM FOR OPTIC DISC DETECTION}

Though there are lot of algorithms for detecting OD location, we have compared Li and Chutatape's Method and Tomi Kauppi and Heikki Kälviäinen with our proposed optic disc detection method since, these two are considered as a baseline reference work and they were already evaluated with the same images by Tomi Kauppi, the main contributor of DIARETDB1.

\subsection{Li and Chutatape's Method}

In $\mathrm{Li}$ and Chutatape's method, the PCA was applied to the optic disc regions extracted from the training set of gray level images to determine the features that characterise the variations of the optic disc appearance [2]. These linearly independent features were referred to as eigendiscs. The method assumed that each input optic disc image can be approximated as a linear combination of the eigendiscs and the mean training set optic disc. If the squared Euclidean distance between a test input image and the approximated test image was small the input image is considered as an optic disc. The squared Euclidean distance was computed in a sliding window on every image point and the point with the minimum distance was chosen to be the optic disc location. In practice, $\mathrm{Li}$ and Chutatape computed the Euclidean distance only on bright candidate areas determined in a preprocessing stage to reduce the computational load. In practice, $\mathrm{Li}$ and Chutatape computed the Euclidean distance only on bright candidate areas determined in a pre-processing stage to reduce the computational load.

\subsection{Colour Decorrelated Templates Based Method}

In the above method PCA is applied to find the vectors that span the colour of the optic disc colour basis and decorrelate the colour channels[1](colour decorrelated template space). Therefore, each channel is now independent and describes colour characteristic of an optic disc. The method is similar to Xie.X and Mirmehdi[5], in which colour channel decorrelation was used as part of a random texture analysis application. Colour data of several optic disc images are extracted from the training images and used to generate the colour decorrelated template space. Before conduction template matching the eigenvectors are used to project test images to the colour decorrelated template space.In each location of the image the similarity between a template and the same sized image patch is measured with the squared Euclidean distance. The input image is considered as an optic disc when the squared Euclidean distance between a test input image and the approximated test image was small.Over every image point the squared Euclidean distance was computed using a sliding window and the point with the minimum distance was considered as the optic disc location.

The manually annotated optic disc locations of the DIARETDB1 database were used for the evaluation of the algorithms. The results from [1] and [2] is compared with the results of the proposed method. The mean optic disc diameter in the training set images was approximately 200 pixels, therefore a $200 \times 200$ template was used for the evaluation basis (for the two compared methods). A threshold distance from the ground truth was varied and the accuracy was the percentage of detected optic discs inside the threshold distance. The distance from ground truth was normalised with the length of optic disc radius (100 pixels).

\section{THE PROPOSED METHOD}

In the proposed method, the OD location will be detected using blue channel of the image of green channel of the image based on the intensity estimate on blue channel of the image. Further, instead of searching all the possible locations of better matching location, the GA will search the entire space in a fast manner by considering all the potential regions.

\subsection{Intensity Estimaion using Blue layer}

The following figure shows the proposed nonlinear optimization technique for OD detection using Blue channel and Green channel Intensity information. 


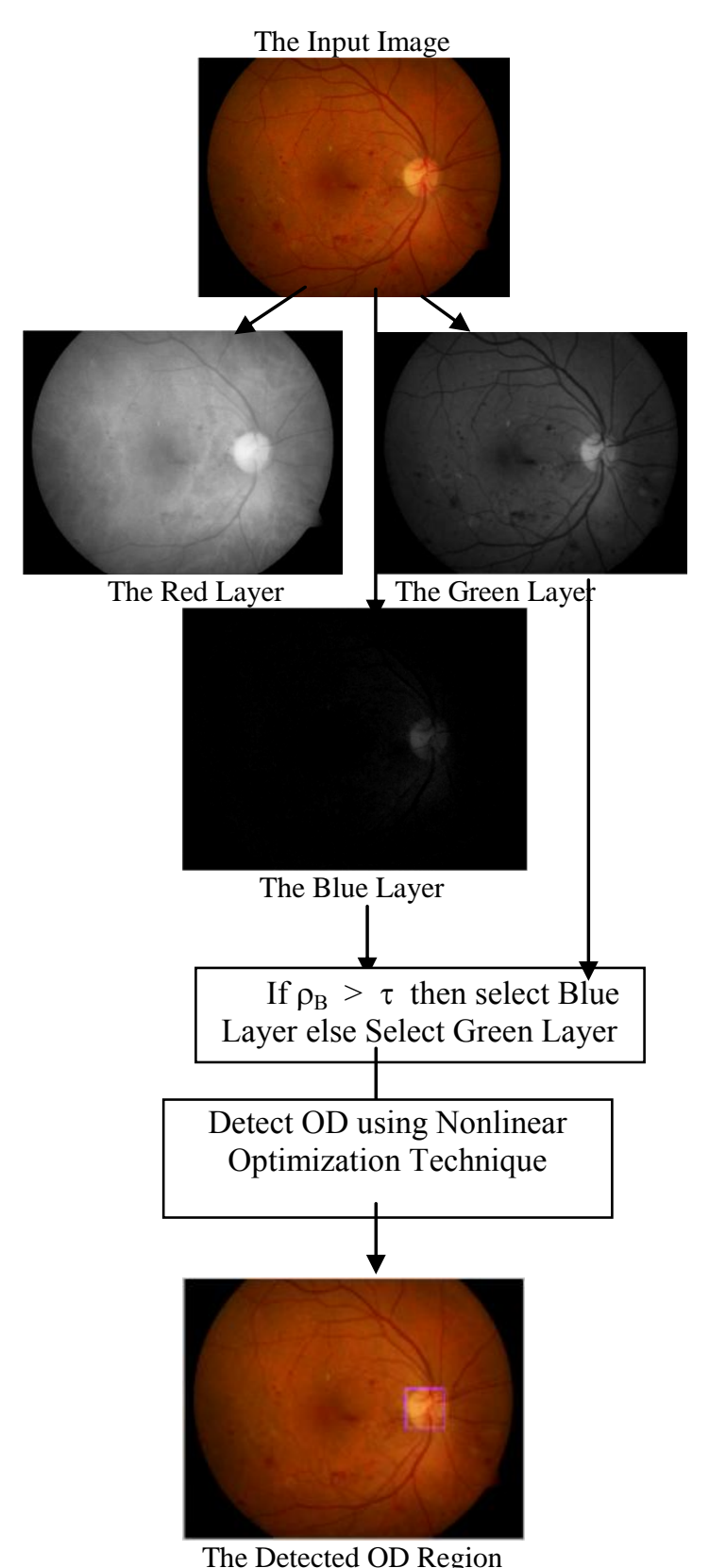

Fig 1: The Color Channel Selection using Blue Channel Intensity Estimate

$$
\rho_{B}=\frac{\sum_{i=1}^{n} \sum_{i=1}^{m} B_{(i, j)}}{(m \times n)}, \forall B_{(i, j)}>0
$$

So the normalized intensity estimate $\rho_{B}$ may have value between 0 and 1 . If it is 0 then it means, all the blue layer pixels are having value equal to 0 . If it is 1 then it means, all the blue layer pixels are having values greater than 0 .

Even though the blue layer pixel will have values near zero, and will not be visible if we try to display that layer. If $50 \%$ percent ot the pixels will have greater than 0 values, then we can use the green layer as the target image to detect the OD else we may use the green layer as target image.

$$
\begin{aligned}
& \text { If } \rho_{\mathrm{B}}>\tau \\
& \text { //set Blue Layer as target Image } \\
& \quad \mathrm{T}=\mathrm{B} \\
& \text { Else } \\
& / / \text { set Green Layer as target Image } \\
& \quad \mathrm{T}=\mathrm{G} \\
& \text { End }
\end{aligned}
$$

In this experiment, we used the threshold $\tau=0.5$ to dicide whether to use blue layer of green layer for detecting the OD.

\subsection{The Design of GA for OD detection}

After selecting the suitable channel by using the above equation 1 , the optimum OD location will be found using GA.

The 4 variables $\mathbf{i}, \mathbf{j}, \mathbf{r}_{\mathbf{1}}$, and $\mathbf{r}_{\mathbf{2}}$, which will decide the optimum location and size of the OD should be represented in GA.

The lower bound of $\mathbf{i}, \mathbf{j}, \mathbf{r}_{\mathbf{1}}$, and $\mathbf{r}_{\mathbf{2}}$ are set as 150,1500 0.The upper bounds i,j, $\mathbf{r}_{1}$, and $\mathbf{r}_{2}$ are set as 1152-150,1500-150 00 where $1152 \times 1500$ is the size of the fundus image in which we are going to find the OD location.

The GA was seeded with the initial population with approximate mid range of these 4 variables as 575,750,5 5 .

The GA applied for detecting the OD will be in the following form. The algorithm will search of optimum location and size of the $\mathrm{OD}$ in the target fundus image $\mathrm{T}$. and will find a OD with in the radius of minumum expected radius $r_{\text {mid }}$ and maximum expected radius $r_{\max }$. On each iteration, the best candidate location is selected based on the intensity estimate given by equation 2 of the fittness function.

\section{THE DIFFERENT ASPECTS OF PROPOSED \\ METHOD}

\subsection{Channel selection based on Intensity Estimate} and $\mathrm{B}$

Let I be the colour fundus image which has three layers R,G

$$
I=\{R, G, B\}
$$

Find the intensity estimate $\rho_{\mathrm{B}}$ at blue layer 


\begin{tabular}{|c|}
\hline Function GA_OD_Detect \\
\hline begin \\
\hline $\begin{array}{l}\text { INITIALIZE population with random candidate solutions; } \\
\text { (Each random candidate solution will represent } 4 \text { variables } i_{j}, r_{l}, \\
\left.\text { and } \boldsymbol{r}_{2}\right)\end{array}$ \\
\hline $\begin{array}{l}\text { EVALUATE each candidate; } \\
\text { (find fitness of each candidate using the fitness function) }\end{array}$ \\
\hline repeat \\
\hline $\begin{array}{l}\text { SELECT parents; } \\
\text { (Select two candidate having best fitness value) }\end{array}$ \\
\hline $\begin{array}{l}\text { RECOMBINE pairs of parents; } \\
\text { (use single point crossover on the selected candidates } \\
\text { and generate new population - this includes the original } \\
\text { parents) }\end{array}$ \\
\hline $\begin{array}{l}\text { MUTATE the resulting children; } \\
\text { (use gaussian mutation on entire population) }\end{array}$ \\
\hline $\begin{array}{l}\text { EVALUATE children; } \\
\quad \text { (find fitness of all new candidates of the population) }\end{array}$ \\
\hline $\begin{array}{l}\text { until TERMINATION-CONDITION is satisfied } \\
\text { end }\end{array}$ \\
\hline $\begin{array}{l}\text { Mark OD on the fundus image using the final optimum values of } \\
\boldsymbol{i}_{\boldsymbol{j}} \boldsymbol{j}, \boldsymbol{r}_{\boldsymbol{l}} \text {, and } \boldsymbol{r}_{\boldsymbol{2}}\end{array}$ \\
\hline
\end{tabular}

\subsection{The Fitness Function}

The following function is used to find the fitness at the location $\mathrm{i}, \mathrm{j}$. The point which has the lowest fitness vaue will be the potential cener of the OD.

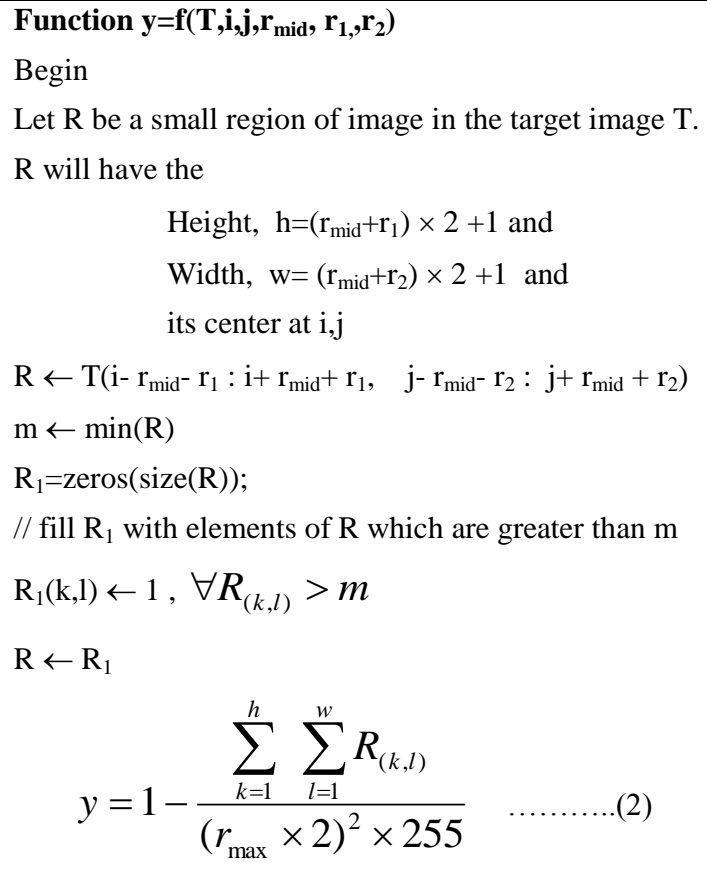

$$
y=1-\frac{\sum_{k=1}^{h} \sum_{l=1}^{w} R_{(k, l)}}{\left(r_{\max } \times 2\right)^{2} \times 255}
$$

End

$$
\begin{aligned}
& \mathrm{T} \text { - is the target image in which we have to locate the } \\
& \text { OD } \\
& \mathrm{i}, \mathrm{j} \text { - the center of the guessed OD location } \\
& \mathrm{r}_{\min }-\text { minumum expected radius of the OD } \\
& \mathrm{r}_{\text {max }} \text {, maximum expected radius of the OD } \\
& r_{1} \text { - the small change in Height } \\
& r_{2} \text { - the small change in width }
\end{aligned}
$$

At the exact location of OD, the above function $y=f\left(T, i, j, r_{\text {mid }}\right.$, $r_{1}, r_{2}$ ) will give the most optimum minimum value.

In this implementation, we set the limit of $\mathrm{i}, \mathrm{j}$ as follows :

$$
\begin{aligned}
& i>150 \text { and } i<(\text { Height of the } T-150) \\
& j>150 \text { and } j<(\text { width of the } T-150)
\end{aligned}
$$

The allowed change in radius $=\mathrm{c}=10$

$$
\begin{aligned}
& r_{\text {mid }=90} \\
& r_{\text {min }}=r_{\text {mid }}-\mathrm{c}=80 \\
& r_{\text {max }}=r_{\text {mid }}+c=100
\end{aligned}
$$

So the algorithm will find a OD of arbitray size between $161 \times 161$ to $201 \times 201$.

We used the Genetic Algorithm tool box of matlab to implement this OD detection algorithm. We set Generations as 20, 'Population Size as 200 and used single point crossover. And for other parameters, the default values of the GA tool box is assumed and different graphs were displayed.

\section{RESULTS AND DISCUSSION}

The manually annotated optic disc locations of the DIARETDB1 database were used for the comparative analysis.

In the following output, the OD region marked by using the groundtruth location information is shown in red the radius is assumed as 100 pixel. The region marked in blue is automatically marked by the proposed algorithm.

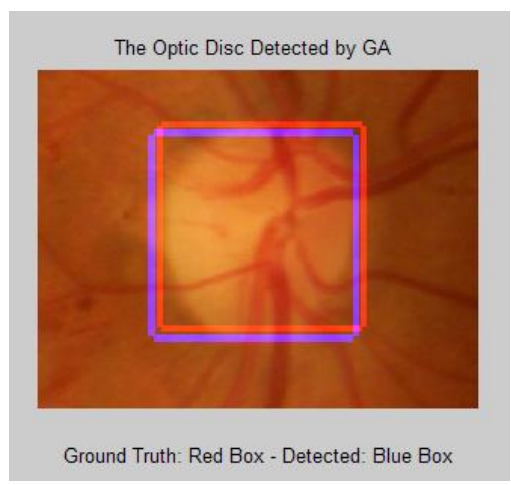

Fig 2: The Marked OD Region

Computation times, accuracy of detection are given in the table 1 of the Annuxure for the first ten database images. The following bar charts were prepared by using the average of that results 


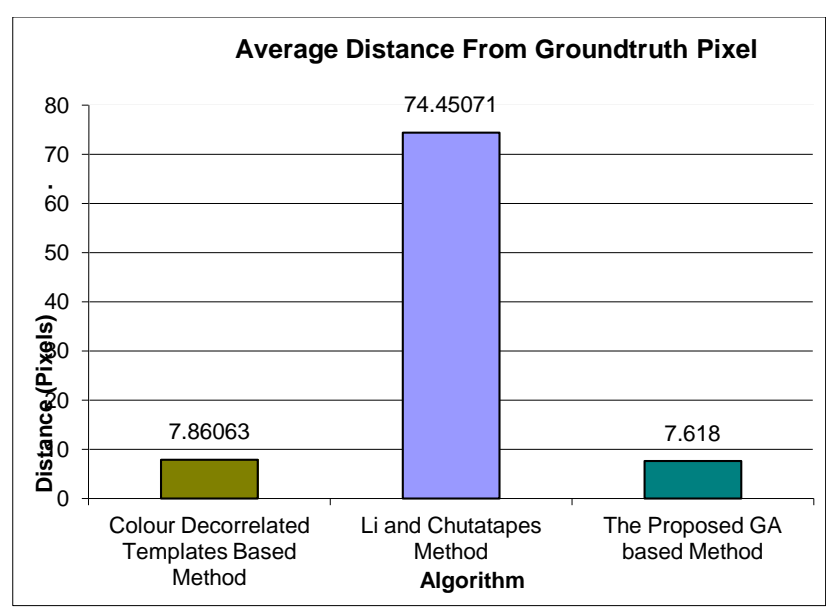

Fig 3: The Average Distance From Groundtruth Pixel

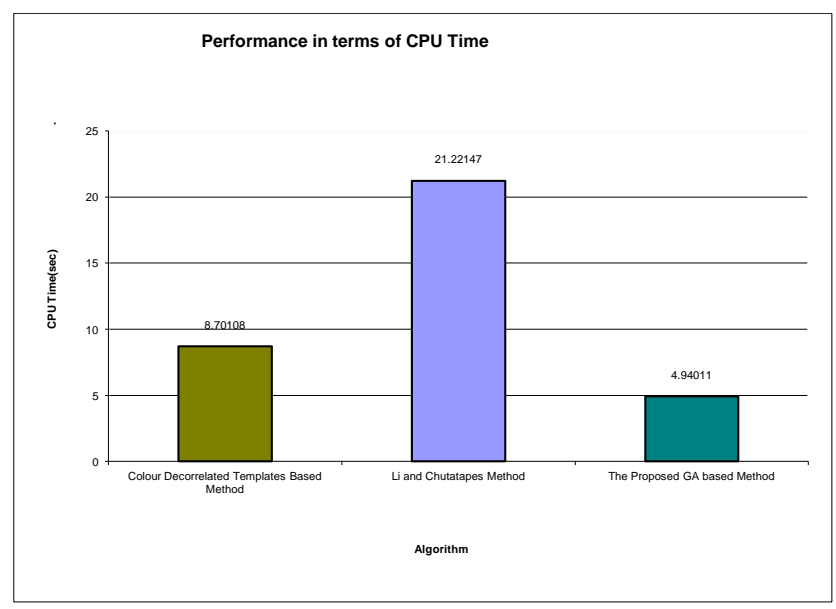

Fig 4: The Performance in terms of CPU time

As shown in the above charts, the proposed GA based method detected the optic disc location in a fundus image in a fast and accurate manner than the other two compared algoritms

The following graphs shows the convergence performance of GA with respect to generations.

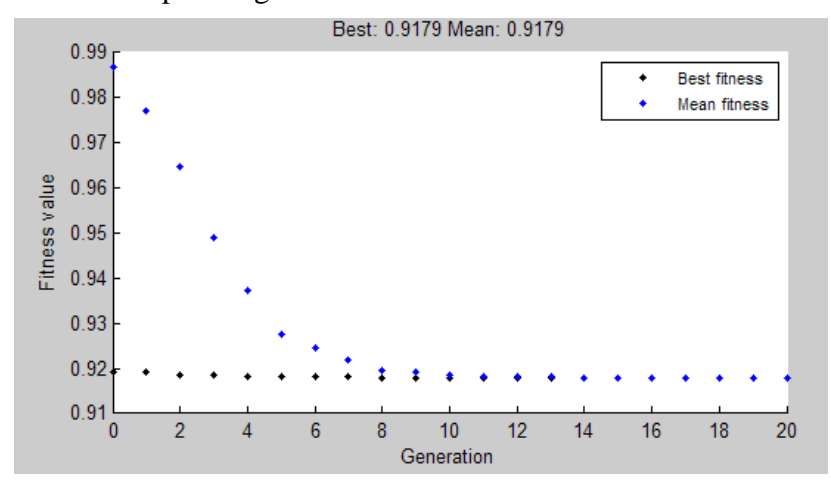

Fig 5: The GA Convergence Performance

As shown in the above graph, the GA based algorithm converges very fast and finds the most optimum location of the OD in fewer generations. We run GA for 20 generation. But the algorithm found the solution at $10^{\text {th }}$ generation. So, in fact, the algorithm was able to find the location in less than 3 seconds.

The score histogram of GA show how the histogram of all the found solutions during all the generations. If we see this histogram, then the bar corresponding to the solution 0.9179 (the highest bar) shows the significance and the confidence of that result. It means, the same result was found as optimum result during more than 150 times during the random GA search process.

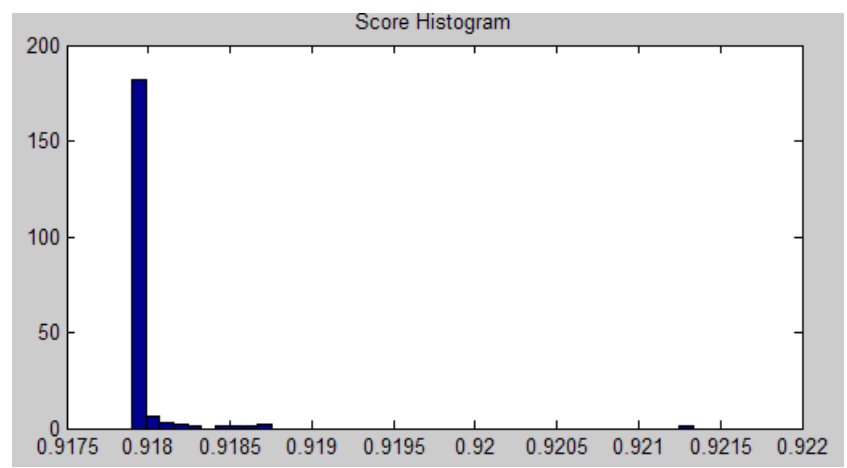

Fig 6: The GA Score Histogram

\section{ADVANTAGES OF PROPOSED METHOD}

It is obvious that the proposed method will only consume lesser time than the above two methods since there is no complex operations such as FFT, PCA and eigenvector calculations involved in it.

Further, the optimization technique will converge very fast since it will randomly chose potential OD locations and will find a optimum location from a limited set of locations. On the other hand, for better results, the other two methods should do the calculations at every possible OD locations/ pixels of the image under consideration from a 1500 x 1152 size fundus image.

In the pre-processing $\mathrm{Li}$ and Chutatape's method[2] used candidate areas having pixels with the highest $1 \%$ gray levels in the intensity image and then removing areas with pixels under a predefined threshold. This restricted the minimum squared Euclidean distance search space and pruned some false optic disc locations. At the same time, the pre-processing steps caused their method to unidentify dark optic discs in the periphery of the eye fundus area resulting high error distances. Computationally, the performance of the colour decorrelated templates[1] was faster than $\mathrm{Li}$ and Chutatape's method. The computation of colour decorrelated template space and template matching with colour decorrelated templates through FFT were very fast to compute and easy to implement. Compared to $\mathrm{Li}$ and Chutatape's method, the computation of the colour decorrelated templates over image involved 3 correlations, whereas their method involved $\mathrm{K}+2$ correlations using the formulation by Turk and Pentland [3]. $\mathrm{K}$ is the number of eigendiscs (eigenvectors).

\section{CONCLUSION}

Most of the previous methods failed in detecting OD in brighter images were the intensity of OD region is almost similar or higher than the several other regions in the target image. But, proposed method was successful in finding OD in fundus image with both brighter and darker image intensities. The GA based search algorithm was very fast in locating the OD location. The accuracy of the detected location was very much depend up on the matching policy. So, the future work may address more efficient matching policies. Since GA is reducing overall search time considerably, we may even consider much complex matching policies to improve the accuracy of location and size of the OD. 
The proposed GA based optic disc detection method is very faster than other two compared methods and is fining the optimum OD location using the intensity on blue and green layer. The proposed method achieved good accuracy and speed. For example, in the image image004 the $\mathrm{Li}$ and Chutatapes Method failed to detect exact OD location because of the poor intensity at the OD region. But, in this case, our proposed method will use blue channel information so that, it will find the exact location more precisely than the other two methods.

In this work, a simple intensity estimation method is used in the fitness function of the GA. In future works, we may consider more complicated matching polity in the fitness function of the GA. Future works will address the ways to improve the pixelwise accuracy of detection.

\section{ANNEXURE}

\subsection{Sample Result of GA}

The following is a Sample Result of GA during each generations with image001 of DIARETDB1

\begin{tabular}{cccc}
\multicolumn{3}{c}{ Generation } & \multicolumn{3}{c}{$\mathrm{f}$-count Best $\mathrm{f}(\mathrm{x})$ Mean $\mathrm{f}(\mathrm{x})$} \\
1 & 200 & 0.9242 & 0.9785 \\
2 & 400 & 0.9242 & 0.9666 \\
3 & 600 & 0.924 & 0.9544 \\
4 & 800 & 0.9232 & 0.9419 \\
5 & 1000 & 0.9229 & 0.9354 \\
6 & 1200 & 0.9222 & 0.9309 \\
7 & 1400 & 0.9206 & 0.9279 \\
8 & 1600 & 0.9187 & 0.9252 \\
9 & 1800 & 0.9181 & 0.9243 \\
10 & 2000 & 0.9179 & 0.9255 \\
11 & 2200 & 0.9179 & 0.9294 \\
12 & 2400 & 0.9179 & 0.9268 \\
13 & 2600 & 0.9179 & 0.9237 \\
14 & 2800 & 0.9179 & 0.9215 \\
15 & 3000 & 0.9179 & 0.9205 \\
16 & 3200 & 0.9179 & 0.9193 \\
17 & 3400 & 0.9179 & 0.9185 \\
18 & 3600 & 0.9179 & 0.9182 \\
19 & 3800 & 0.9179 & 0.918 \\
20 & 4000 & 0.9179 & 0.9179
\end{tabular}

Optimization terminated: maximum number of generations exceeded.

The following is the final result of GA with image001 of DIARETDB1

Total Consumed Time

$: 4.3362 \mathrm{sec}$

The number of generations was $\quad: 20$

The number of function evaluations was $\quad: 4200$

The best function value found was $\quad: 0.917897$

The Optic Disc Center

$:(554,1215)$

Estimated $\mathrm{X}$ radius $\quad: 90$ pix

Estimated $\mathrm{Y}$ radius : 90 pix

The Difference Between Original

and Detected Optic Disc Center

: 5.39 pix 


\subsection{Results With Three Different Methods}

In the following table, The columns of Method I and Method II contains original results from Colour Decorrelated Templates Based Method and Li and Chutatapes Method the column Method III contains results of GA based method proposed in this work.

Table 1. Computation time evaluation results for the ten first database images and distances between detected optic discs and ground truths in pixels. (Distances over optic radius(100pixels) Marked with black).

\begin{tabular}{|c|c|c|c|c|c|c|}
\hline \multirow[b]{2}{*}{$\begin{array}{l}\text { DIARETDB } 1 \\
\text { Image }\end{array}$} & \multicolumn{2}{|c|}{$\begin{array}{c}\text { Method I } \\
\text { Colour Decorrelated Templates Based } \\
\text { Method }\end{array}$} & \multicolumn{2}{|c|}{$\begin{array}{c}\text { Method II } \\
\text { Li and Chutatapes Method }\end{array}$} & \multicolumn{2}{|c|}{$\begin{array}{c}\text { Method III } \\
\text { The Proposed GA based OD } \\
\text { Detection Method }\end{array}$} \\
\hline & $\begin{array}{l}\text { Distance from } \\
\text { Groundtruth } \\
\text { (pixels) }\end{array}$ & $\begin{array}{l}\text { Computation } \\
\text { Time(s) }\end{array}$ & $\begin{array}{l}\text { Distance from } \\
\text { Groundtruth } \\
\text { (pixels) }\end{array}$ & $\begin{array}{l}\text { Computation } \\
\text { Time(s) }\end{array}$ & $\begin{array}{l}\text { Distance from } \\
\text { Groundtruth } \\
\text { (pixels) }\end{array}$ & $\begin{array}{c}\text { Computation } \\
\text { Time(s) }\end{array}$ \\
\hline image001 & 9.4868 & 9.3874 & 10.1980 & 21.6208 & 5.39 & 4.9071 \\
\hline image002 & 6.7082 & 8.9209 & 10.4403 & 18.2414 & 6.08 & 4.9772 \\
\hline image003 & 15.5242 & 8.6041 & 11.1803 & 19.0500 & 8.54 & 4.8770 \\
\hline image004 & 15.5242 & 8.6787 & 654.7648 & 33.3511 & 8.60 & 4.7769 \\
\hline image005 & 2.0000 & 8.4736 & 14.0357 & 18.7710 & 9.85 & 5.1073 \\
\hline image006 & 14.0000 & 8.5706 & 10.7703 & 18.8342 & 8.06 & 5.2676 \\
\hline image007 & 2.2361 & 8.4846 & 8.0623 & 26.9084 & 7.07 & 4.7368 \\
\hline image008 & 2.8284 & 8.7475 & 9.0554 & 18.0940 & 4.12 & 4.8069 \\
\hline image009 & 8.0623 & 8.6124 & 6.0000 & 18.4484 & 7.07 & 4.9671 \\
\hline image 010 & 2.2361 & 8.531 & 10.0000 & 18.8954 & 11.40 & 4.9772 \\
\hline Average & 7.8606 & 8.7011 & 74.4507 & 21.2215 & 7.618 & 4.94011 \\
\hline Accuracy & \multicolumn{2}{|c|}{$100 \%$} & \multicolumn{2}{|c|}{$90 \%$} & \multicolumn{2}{|c|}{$100 \%$} \\
\hline
\end{tabular}

\section{REFERENCES}

[1] Tomi Kauppi, Heikki Kälviäinen, "Simple and Robust Optic Disc Localisation Using Colour Decorrelated Templates", Advanced Concepts for Intelligent Vision Systems, 10th International Conference, ACIVS 2008, Juan-les-Pins, France, October 20-24, 2008.

[2] Li, H., and Chutatape, O. Automatic location of optic disk in retinal images. In Proceedings of the International Conference on Image Processing (ICIP2001) (Thessaloniki, Greece, 2001), vol. 2, pp. 837-840.

[3] Turk, M. A., and Pentland, A. P. Eigenfaces for recognition. Journal of Cognitive Neuroscience 3, 1 (1991), 71-86.

[4] Jaspreet Kaur and Dr.H.P.Sinha, "Automated Localisation of Optic Disc and Macula from Fundus Images", International Journal of Advanced Research in Computer Science and Software Engineering Volume 2, Issue 4, April 2012

[5] Xie, X., and Mirmehdi, M. Localising surface defects in random colour textures using multiscale texem analysis in image eigenchannels. In Proceedings of the International Conference on Image Processing (ICIP2005) (Genova, Italy, 2005), vol. 3, pp. 837-840.Xie, X., and Mirmehdi, M. Localising surface defects in random colour textures using multiscale texem analysis in image eigenchannels. In Proceedings of the International Conference on Image Processing (ICIP2005) (Genova, Italy, 2005), vol. 3, pp. 837-840.

[6] Meindert Niemeijer, Bram van Ginneken, Stephen R. Russell, Maria S.A. Suttorp-Schulten,MichaelD.Abràmoff,
"Automated Detection and Differentiation of Drusen, Exudates, and Cotton-Wool Spots in Digital Color Fundus Photographs for Diabetic Retinopathy Diagnosis",Investigative Ophthalmology and Visual Science, Vol.48, pp.2260-2267, 2007.

[7] Dr.lee Kai Yuen(private practice) Tsang CY Luke, Lam Hon Man,et.al.(Family Medicine Service,Department of Health,HKSAR)

[8] C.Jayakumari and T.Santhanam, "Detection of Hard Exudates for Diabetic Retinopathy Using contextual Clustering and Fuzzy Art Neural Network", Asian Journal of Information technology, Vol.6,No8,pp.842-846,2007.

[9] C.Jayakumari and T.Santhanam,"An intelligent approach to detect hard and soft exudates using echo state neural network", Information Technology Journal 2008.

[10] Emily, Y. 2003. Diabetic Retinopathy, American academy of . ophthalmology - Retina panel, Preferred practice patterns

[11] Automatic detection of hard and soft exudates in fundus images using color histogram thresholding. S.kavitha, K.Duraisamy, European Journal of scientific research.

[12] Liu, Z., Chutatape, O. and Krishnan, S. M. 1997.Automatic image analysis of fundus photograph.Proceedings of 19th Annual Int. Conf. IEEE Engineering in Med. and Biology Society. vol. 2, 524-525.

[13] Automatic Exudates detection in diabetic retinopathy images using digital image processing algorithm. P.Aravindhan, 
P.N.Jebarani sargunar, proceedings on information science and applications ICISA 2010, Chennai, India.

[14] "DIARETDB1 - Standard Diabetic Retinopathy Database Calibration level 1

[15] Akara Sopharak, Bunyarit Uyyanonvara, Sarah Barman, "Automatic Exudate Detection from Non-dilated Diabetic Retinopathy Retinal Images Using Fuzzy C-means
Clustering", Journal of Sensors, Vol.9, No.3, pp.2148-2161, 2009.

[16] Gonzalez .R and R.Woods 1993 Digital Image Processing, Addison - Wesley press.

[17] Madhuri A.Joshi, Digital Image Processing, An algorithmic Approach 